\title{
The treatment of scabies
}

\section{Commens, Department of Dermatology, Westmead Hospital, University of Sydney, Sydney}

\section{SYNOPSIS}

Most experienced clinicians will miss the diagnosis of scabies at least once. As a result the patient's itch and discomfort will be prolonged and additional contacts needlessly infected. Scabies should be excluded in any patient with itch. The history, particularly itching of recent onset, and careful scrutiny of hands and wrists will usually establish the diagnosis. Scabies can be confirmed with skin scrapings. Treatment is effective provided it is done scrupulously. Permethrin cream is preferred in most cases, but severe cases may require oral ivermectin.

Index words: itch, infestations, permethrin, ivermectin.

(Aust Prescr 2000;23:33-5)

\section{Introduction}

Scabies is caused by Sarcoptes scabiei, a barely visible mite that is host specific for humans. Sarcoptes mites from other animals may also occasionally infest humans.

Mite movement is temperature dependent. The mite is almost immobilised below $20^{\circ} \mathrm{C}$. Transmission of mites between humans is therefore increased in a warm environment. Human to human contact of about 20 minutes allows the mite to transfer. Transmission also occurs via contaminated clothing, bedding, furniture and contaminated epithelial debris shed from scabetic patients.

The intraepidermal burrow is the pathognomonic clinical sign of scabies and contains the pregnant female mite, eggs, faeces and other discarded material from the mites. The eggs hatch in two weeks. The larvae form intraepidermal lesions whilst they mature into the adult form. This maturation takes only 2-3 weeks. Most humans develop a stable population of between 10-50 pregnant females in an ongoing infestation. Some forms of scabies have thousands of mites and are highly contagious.

\section{Clinical settings}

Scabies in the family setting usually means one or two other members of the family will also be affected. These patients usually have a low mite count (10-50) and appropriate treatment of the whole family is curative. In extended families, contact tracing is important to make sure that relatives and other people who had significant physical contact with the infested patient are also treated.

Scabies in a nursing home or institution is a difficult problem. The patients may be disabled, immobile or otherwise compromised and often have high mite counts. As a result they are more infectious. Nursing staff and patients in the surrounding areas will often become infected. Minor epidemics then result. The scabies may become long-standing despite treatment. Extensive contact tracing and treatment of affected patients and the immediate environment are therefore important.

Some patients develop severe scaling and crusting as a result of the infestation and have tens of thousands of mites. This has been described as Norwegian scabies (probably because it was first reported in Norway in 1848 when it was thought to be an endemic form of leprosy). Discarded crust and scale in bedding has hundreds of potentially infectious mites. Treating the patients' environment is particularly important.

\section{Clinical features}

Scabies should be considered in any patient with an unexplained itch of recent onset (Table 1). A definite diagnosis can be made with recognition of one major criterion (Table 2) and a likely diagnosis can be made with two or more of the minor diagnostic criteria.

\section{Itch}

Itch is the predominant feature in all but the most compromised patients. The itchy rash begins about four weeks after the beginning of the infestation coinciding with sensitisation. It is often worse at night.

\section{Table 1}

When should I think about scabies?

Unexplained pruritus especially if other contacts are also itchy

'Atopic or irritant dermatitis' of very recent onset

Persistent insect bite reactions

Recurrent impetigo with itch

Pustular lesions on the palms and soles particularly in the young Unusual urticaria

Unusual pruritic psoriasiform rashes that are crusted and scaling or blistering

\section{Table 2}

\section{Diagnosis}

Major diagnostic criteria (presence of one confirms diagnosis)

- Identifiable typical burrow particularly associated with itchy rash

- Positive skin scrapings showing eggs or mite or faeces

Minor criteria (two needed for likely diagnosis)

- Typical itchy rash

- Sudden onset of unexplainable itchy rash

- Contact with a scabetic patient

- Papules on penis 
Wrist showing lesions and intraepidermal burrow of the scabies mite

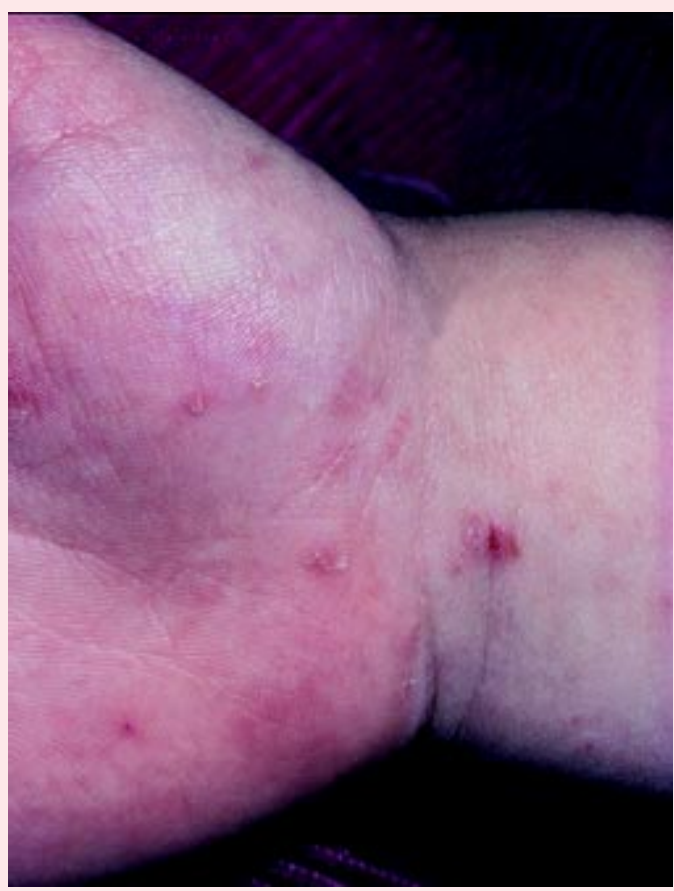

The itch is due to a combination of non-specific factors and specific immunological events as patients develop hypersensitivity to the mite and its products. A prickling irritation can be felt when mites move around on the warm skin.

Secondary dermatitis is widespread due to these immunological mechanisms, scratching and the irritation caused by the treatments applied by an increasingly desperate itchy patient.

\section{Burrows}

The diagnostic sign of scabies is the burrow (see picture). This is an intraepidermal track made by the egg laying female and is usually less than a centimetre long. The opening of the burrow may have a mild scale while the blind end contains the female. Most adult humans will have a burrow on the hands or wrists. Children will often have burrows on the feet as well. Burrows may also be found at other sites.

\section{Other presentations}

Atypical clinical features are less common. Patients may develop urticarial or blistering rashes if they are very hypersensitive to the mite. Nodular lesions may develop from chronic scratching and infestation. Children may have a pustular eruption particularly around their hands and feet.

\section{Investigations}

In most patients, scraping a number of burrows will reveal the mite, eggs or faeces. This confirms the diagnosis and is a major diagnostic criterion (Table 2).

\section{Treatment}

There are some general principles (Table 3), but the treatment is influenced by the clinical setting.

\section{Table 3}

\section{General strategies in scabies management}

Confirm the diagnosis preferably by identifying a typical burrow or positive skin scrapings.

Trace all contacts and ensure appropriate treatment.

Co-ordinate treatment.

Apply antiscabetic cream thoroughly. This usually means the entire body from the neck down. In some individuals the head must also be treated. Repeat in one week.

Treat all contaminated clothing and bedding. In some circumstances the immediate environment may also need decontamination.

Follow up 4-6 weeks later to ensure clearance.

\section{Table 4}

\section{Treatment of household scabies}

- Treat the whole family simultaneously, even members who are not itchy. The initial strategy is to kill the mite. Once this is achieved then treatment for the itch and dermatitis can begin.

- Apply the acaricidal preparation to dry skin from the neck down emphasising treatment of all sites - e.g. under nails, soles of feet, natal cleft. Permethrin cream is a good primary treatment and the author advises patients to leave this on for 24 hours. The head requires treatment if involved. This should be looked for in the elderly, infants, compromised patients and those with Norwegian scabies.

- Family members with likely scabies should have a repeat permethrin treatment in one week.

- Wash all clothes and bed sheeting being used in hot water and either iron or put through a hot dryer. An alternative is to store bedding and clothing for 1-2 weeks.

- Advise patients that 'mite killing' cream will not immediately resolve the itch or the rash.

- Begin antipruritic dermatitis treatment immediately after each course of antiscabetic creams:

(a) moisturise before and after showering

(b) avoid irritants such as too much soap and excessive sweating

(c) apply appropriately potent topical corticosteroids to the rash for the next $2-3$ weeks

(d) treat secondary infection

\section{Family (Table 4)}

An inviolable principle of scabies treatment is to treat all significant contacts. In practice this means all members of the family should have at least one treatment. Patients with definite or probable scabies should have two treatments. Caution should be exercised in treating infants, pregnant or lactating women, and the very elderly, as some preparations may be more toxic.

The treatment choice rests largely between topical permethrin or lindane applied to the skin. Permethrin is preferred because of its apparent lesser toxicity. A large number of other agents may be used in special circumstances (Table 5). The application must be done scrupulously. If one burrow is spared then the infestation will persist. The cream therefore needs to be massaged under nails and reapplied to any areas that are washed. 


\begin{tabular}{|c|c|}
\hline \multicolumn{2}{|l|}{ Antiscabetic treatment } \\
\hline Treatment & Comment \\
\hline Permethrin & $\begin{array}{l}\text { A synthetic pyrethroid which is } \\
\text { probably the safest antiscabetic } \\
\text { treatment. Proven effectiveness. } \\
\text { Probably safe in infants, pregnant } \\
\text { and lactating women, and the elderly. }\end{array}$ \\
\hline Benzyl benzoate & $\begin{array}{l}\text { This is often irritating. Toxicity is } \\
\text { uncertain. It can be used as a spray for } \\
\text { furnishings and the environment } \\
\text { where there is heavy contamination. }\end{array}$ \\
\hline $\begin{array}{l}\text { Lindane } \\
\text { (gamma benzene } \\
\text { hexachloride) }\end{array}$ & $\begin{array}{l}\text { The potential for neurotoxicity } \\
\text { limits the use of this agent particularly } \\
\text { in infants, pregnant and lactating } \\
\text { women, and in the elderly. }\end{array}$ \\
\hline Ivermectin & $\begin{array}{l}\text { Single dose may be effective. } \\
\text { Simultaneous topical treatment is } \\
\text { optional. Sometimes repeated doses } \\
\text { are necessary. This drug is an } \\
\text { important development in treating } \\
\text { compromised patients, crusted } \\
\text { Norwegian scabies, widespread } \\
\text { unresponsive scabies and possibly } \\
\text { some community epidemics in nursing } \\
\text { home situations. Before prescribing, } \\
\text { medical practitioners should be aware } \\
\text { of the potential adverse effects and } \\
\text { controversies in treating the young or } \\
\text { the very elderly on multiple } \\
\text { medications. }\end{array}$ \\
\hline $\begin{array}{l}\text { Miscellaneous other agents } \\
\text { including maldison, } \\
\text { and } 6 \% \text { precipitated } \\
\text { sulphur cream }\end{array}$ & $\begin{array}{l}\text { These agents require specialised } \\
\text { experience and are not recommended } \\
\text { as first line treatment. Some of } \\
\text { these may also be used for spraying } \\
\text { furniture. }\end{array}$ \\
\hline
\end{tabular}

\section{Nursing homes and institutions (Table 6)}

All cases in a nursing home must be identified and an assessment made of all their contacts who would be likely to be infested. This often means that 100 or more people may need assessment and counselling. There are protocols available from area health boards that spell out the importance of co-ordinated treatment.

Affected patients in nursing homes may have severe contractures and be compromised. Applying the antiscabetic agent thoroughly may be difficult. Failure to cure an epidemic may be because of a persistent, highly infectious patient or unrecognised contacts particularly amongst staff. All suspected patients should have two applications of topical antiscabetics, in the author's opinion. Those with high mite counts or persisting scabetic infections may require additional treatment with ivermectin (Table 5).

\section{Norwegian scabies}

Patients with severe scaling and crusting or who are very immunocompromised are highly infectious and are often difficult to cure with topical preparations. Ivermectin is indicated. Importantly, the patients' environment must be sterilised including bedding, chairs, carpets and curtains.

\section{Table 6}

\section{Treatment of institutional scabies and those who are} highly infectious

- Local Area Health Board can usually provide a scabies control protocol. Co-ordinated treatment is essential if patients belong to different general practitioners.

- Treat all infectious patients with stringent isolation procedures until cured. Multiple treatments may be necessary and consider adjunctive oral treatment such as ivermectin.

- Treatment of the entire body may be necessary including the head. Patients with Norwegian scabies will need exfoliation creams (e.g. salicylic acid 6\% cream), and consultation with a dermatologist is recommended.

- All significant contacts should be evaluated with careful and, if appropriate, a full body examination. Scabies in affected staff, patients and contacts can look atypical. If possible the diagnosis should always be confirmed with microscopic scrapings or identification of typical burrows.

- Treat all affected contacts simultaneously.

- Nursing staff or attending carers should wear plastic disposable gloves and practise strict barrier nursing. They should be careful about excessive shaking of bedding and clothing that may disperse infected scale and debris widely into the environment.

- An acaricidal spray (e.g. benzyl benzoate) will help to decontaminate rooms with application of the spray to bedding, chairs, floors, pillows. Fumigation and closure of the area are other options.

- Shoes should be placed in a plastic bag for two weeks. Clothing and sheeting should be washed in hot water and ironed or put through a hot dryer. Storage for two weeks may be necessary for items that cannot be decontaminated.

- Follow-up with weekly treatments to highly infected individuals is important. Repeat scrapings may be necessary to ensure that these patients are finally clear.

- Contacts must be followed up at 4-6 weeks. Ensure that staff, relatives and contacts are fully aware of the implications of scabies and are warned to be suspicious of persistent or recurrent itchy rashes for the next few months.

\section{Self-test questions}

The following statements are either true or false (answers on page 47)

3. Norwegian scabies is not present in Australia.

4. The itch of scabies resolves within 24 hours of successful antiscabetic treatment.

\section{New National Medicines Policy}

The 2000 National Medicines Policy has been launched. Copies are available from the Department of Health and Aged Care: fax 026289 7746, phone 0262897491. 